\title{
Microwave-Driven SET-Box with Superconducting Island
}

\author{
A. Hädicke and W. Krech \\ Institut für Festkörperphysik, Friedrich-Schiller-Universität Jena, Max-Wien-Platz 1 \\ D-07743 Jena, Germany
}

(June 1994)

\begin{abstract}
The effect of a high-frequency microwave irradiation on the averaged stationary charge in a single-electron tunneling box (SET-box) with superconducting island is investigated. The resulting $\langle Q\rangle-Q_{g}$ characteristics are modified compared with those from the well-known constant voltage-driven box. It can be shown that there is a cross-over from the 2e-periodic regime in $Q_{g}$ corresponding to the parity effect to 1e-periodic behavior not only with increasing temperature but also with increasing irradiation amplitude.
\end{abstract}

Typeset using REVTEX 


\section{INTRODUCTION}

The tunneling of single electrons (SET) through ultrasmall junctions has become of much experimental and theoretical interest (see e.g. [1])). The main conditions to have charging effects (Coulomb blockade) are low temperatures $\left(k_{B} T \ll E_{C}\right)$ and large tunnel resistances $\left(R_{T} \gg R_{Q}=h / e^{2}=25.8 \mathrm{k} \Omega\right)$. This guarantees that single-electron effects are lifted out of thermal and quantum fluctuations. $e$ and $h$ are the elementary charge and Planck's constant, respectively, $k_{B}$ is Boltzmann's constant. $E_{C}$ denotes the relevant Coulomb energy under consideration.

We consider the so called SET-box formed by a SET junction and a capacity $C_{s}$ in series as described in Fig. 1. It is known that the mean electric charge on the island of this SET-box can be increased in integer steps by a continuous increase of the external voltage $V=V_{o}$ (see e.g. [2]) corresponding to an increase of the charge $Q_{g}$ at the capacity $C_{s}\left(Q_{g}=C_{s} V_{o}\right)$. The staircase function $\langle Q\rangle$ vs. $Q_{g}$ is the sharper the temperature is lower (see Fig. 2). The tunnel junction is characterized by the capacity $C$ and the imaginary part of the quasi-particle current amplitude $\operatorname{Im} I_{q}$ which is related with the phenomenological tunnel resistance $R_{T}$.

A new aspect which has thoroughly been investigated as well theoretically and experimentally are parity effects on the superconducting island [3-11]. It has been observed that the parity based $2 \mathrm{e}-$ periodicity of the charge characteristics disappears in the vicin-

ity of a cross-over temperature which is much lower than the critical temperature of the superconductor.

There are some articles dealing with time-dependent phenomena in mesoscopic tunnel junctions [12-16]. We have investigated in an earlier paper [17 the influence of a microwave irradiation on the stationary behavior of a normalconducting double-junction.

The aim of this paper is to investigate the influence of a microwave irradiation on SET-box parity effects in stationary regime. Because of the fact that in some sense the 
microwave irradiation acts similarly like temperature increase the cross-over mentioned above can also be achieved by a sufficient strong irradiation.

\section{NORMALCONDUCTING BOX}

For a normalconducting island the relevant energy function which enters the transition rates reads as

$$
E_{n}^{c h}\left(Q_{g}\right)=\frac{e^{2}}{2\left(C+C_{s}\right)}\left(n-Q_{g} / e\right)^{2}
$$

$n$ labels the number of electrons on the island. The index "ch" means that this energy is the charging energy. The charge $Q_{g}$ at the capacity $C_{s}$ is connected with the external voltage $V_{o}$ via the relation

$$
Q_{g}=V_{o} C_{s}
$$

The master equation describing the dynamics of the occupation probabilities of the SET-box reads as 18,19

$$
\frac{d}{d t} \sigma_{n}(t)=-\left(\Gamma_{n-1, n}+\Gamma_{n+1, n}\right) \sigma_{n}(t)+\Gamma_{n, n+1} \sigma_{n+1}(t)+\Gamma_{n, n-1} \sigma_{n-1}(t)
$$

The rates $\Gamma_{n \pm 1, n}$ which depend on the energy differences (cf. Eq. (1) , $E_{C}=e^{2} /\left(2\left(C+C_{s}\right)\right)$ )

$$
\Delta E_{n \pm 1, n}^{c h}\left(Q_{g}\right)=E_{n \pm 1}^{c h}\left(Q_{g}\right)-E_{n}^{c h}\left(Q_{g}\right)=E_{C}\left(1 \pm 2 n \mp 2 Q_{g} / e\right)
$$

are given by

$$
\Gamma_{n \pm 1, n}\left(Q_{g}\right)=\frac{1}{e} \operatorname{Im} I_{q}\left[-\Delta E_{n \pm 1, n}^{c h}\left(Q_{g}\right) / \hbar\right]\left\{1-\exp \left[\Delta E_{n \pm 1, n}^{c h}\left(Q_{g}\right) / k_{B} T\right]\right\}^{-1} .
$$

$I_{q}$ are the quasi particle current amplitudes of the NIN junction. The rates $\Gamma_{n \pm 1, n}$ correspond to the tunneling processes connected with the charge transitions $n \rightarrow n \pm 1$, 
respectively. The succession of indices of the rates is the same as for the energy differences, the first index refers to the final state and the second to the initial state. The mean stationary charge in the box is given by

$$
\langle Q\rangle=e \sum_{n} n \bar{\sigma}_{n}\left(Q_{g}\right)
$$

where $\bar{\sigma}_{n}$ are the stationary solution of Eq. (2). They can be written as 20,21

$$
\bar{\sigma}_{n}\left(Q_{g}\right)=\frac{1}{\mathcal{N}} \prod_{m=-\infty}^{n-1} \Gamma_{m+1, m}\left(Q_{g}\right) \prod_{m^{\prime}=n+1}^{\infty} \Gamma_{m^{\prime}-1, m^{\prime}}\left(Q_{g}\right) .
$$

$\mathcal{N}$ guarantees the correct normalization. It can be shown that the mean charge (5) is given by a Boltzmann distribution [2]

$$
\langle Q\rangle=e \frac{\sum_{n} n e^{-E_{n}^{c h}\left(Q_{g}\right) / k_{B} T}}{\sum_{n} e^{-E_{n}^{c h}\left(Q_{g}\right) / k_{B} T}} .
$$

This Boltzmann distribution shows that the charge in the box corresponds to a stationary state and dynamical properties expressed by the current amplitudes do not play any role.

\section{NORMALCONDUCTION BOX WITH IRRADIATION}

The influence of a microwave irradiation on single-electron tunneling through singlejunctions has been studied in Refs. [13-16]. The microwave irradiation is described by an additional oscillating voltage part

$$
V(t)=V_{o}+V_{1} \cos \bar{\omega} t
$$

In this way the microwave field is treated classically neglecting possible back reactions due to tunneling. This is reasonable because all calculations are only done in first order perturbation theory. The effect of a microwave irradiation in case of the box is very similar to that of the double-junction [17] because there is the island which can only be charged in integer quantum units. 
But now the time averaged charge does not more satisfy a Boltzmann distribution. It is rather given by formula (5) where the transition rates have been modified (cf. Eq. (四)) $\Gamma \longrightarrow \bar{\Gamma}$. The new rates read as

$\bar{\Gamma}_{n \pm 1, n}\left(Q_{g}\right)=\frac{1}{e} \sum_{r} J_{r}^{2}(a) \operatorname{Im} I_{q}\left[-\Delta E_{n \pm 1, n}^{c h}\left(Q_{g}\right) / \hbar-r \bar{\omega}\right]\left\{1-e^{\left(\Delta E_{n \pm 1, n}^{c h}\left(Q_{g}\right)+r \hbar \bar{\omega}\right) / k_{B} T}\right\}^{-1}$.

The parameter $a$ is given by

$$
a=\frac{e V_{1}}{\hbar \bar{\omega}}
$$

and $J_{i}$ are the Bessel functions of the first kind. The description via a Boltzmann distribution fails because the irradiation does not allow a pure stationary description. Also the stationary parts of the occupation probabilities carry a relic of the dynamics. From the mathematical point of view the reason is the sum structure of the rates which does not allow a factorization. This approach is based on the assumption that possible measurements of the island charge (e.g. by a on-chip SET-electrometer) would only detect the time-averaged charge. Therefore, these rates correspond already to an averaging with respect to the oscillations. But nevertheless they contain information from the irradiation. For vanishing $\mathrm{AC}$ amplitudes these expressions lead to the ordinary box transition rates without irradiation (田).

The result is (see Fig. 3) that the increase of the $\mathrm{AC}$ voltage amplitude gives rise to a smoothing of the mean charge function compared with that for $V_{1}=0$. In this way the oscillating driving voltage smears out the hard staircase and acts similarly like a temperature increase. This can be demonstrated in the following manner. For $T=0$ the rates without irradiation are nonvanishing only for $\Delta E_{\ldots}^{c h}<0$. But $T>0$ there are also transitions if $\Delta E_{\ldots}^{c h}>0$. In case of irradiation and $T=0$ the condition for nonvanishing rates is $\Delta E_{\ldots}^{c h}+r \hbar \bar{\omega}<0$ which means that there are also transitions for $\Delta E_{\ldots}^{c h}>0$. The comparison of Figs. 2 and 3 shows that there is nearly no difference for a specific choice of parameters. The mechanism can be understood as photon assisted tunneling via excited 
states. The main effect is due to 1 -photon processes $(r= \pm 1)$ because multiple photon processes are strongly damped. But the specific shape of the charge function depends on a complicated interplay of the parameters $a, \bar{\omega}$ and $T$.

\section{SUPERCONDUCTING BOX}

The phenomenological treatment of the superconducting case starts with the observation that the energy content of the island depends on the parity $P_{n}$ of the electron number $n$ on it. This means that for odd $n$ there is the additional energy gap $\Delta$ in the energy function otherwise there is no gap for even $n, P_{n}=n \bmod 2$. This corresponds to the substitution

$$
E_{n}^{c h}\left(Q_{g}\right) \longrightarrow E_{n}\left(Q_{g}\right)=E_{n}^{(c h)}\left(Q_{g}\right)+\Delta \cdot P_{n}
$$

The same approach as in Sec. (III) can be done using the energy function (10). This consideration is correct for zero temperature. Then the charge in the box jumps to the next integer value at the crossing point of two neighbouring $E_{n}\left(Q_{g}\right)$ parabolas (see Fig. 4). The shape of the resulting charge staircase function depends on the relation $\lambda$ between the energy gap $\Delta$ and the Coulomb energy $E_{C}\left(\lambda=\Delta / E_{C}\right)$ (see Fig. 5). The $\langle Q\rangle-Q_{g}$ characteristics shows for $\lambda>0$ a $2 \mathrm{e}-$ periodic behavior in $Q_{g}$. But this approach cannot be correct for $T>0$ because it does not explain the cross-over from the $2 \mathrm{e}-$ periodicity to $1 \mathrm{e}^{-}$ periodicity at a cross-over temperature $T^{*}$ known from experiments [4]. This cross-over arises due to a temperature dependent difference of the free energies $\delta F(T)$ of the island in the even and odd state [4, 11]. $\delta F$ is reduced from $\Delta$ at $T=0$ by entropy contributions. It is not an effect of the temperature dependence of $\Delta$ itself because this temperature is still much lower than the critical temperature $T_{C}$. Therefore $\Delta=\Delta(0)$ throughout this paper. A first order approximation for $\delta F(T)$ reads as

$$
\delta F(T) \approx \Delta-k_{B} T \ln N_{e f f}
$$


$N_{\text {eff }}$ is the effective number of states avaiable for excitation. It depends only weakly on temperature

$$
N_{e f f}=2 N_{(n)}(0) \mathcal{V} \Delta e^{\Delta / k_{B} T} K_{1}\left(\Delta / k_{B} T\right)
$$

$N_{(n)}(0)$ is the normal density of states at the Fermi level and $\mathcal{V}$ corresponds to the island volume. $K_{1}$ labels the modified Bessel function. The cross-over temperature is defined by

$$
\delta F\left(T^{*}\right)=0
$$

For their specific sample Tuominen et al. [4] found

$$
\delta F(T) \approx \Delta\left(1-\frac{T^{*}}{T}\right)
$$

with $T^{*} \approx 300 m K$ much less than $T_{C}$. Of course this is reasonable only for $0 \leq T \leq T^{*}$, the function $\delta F(T)$ is positive definite. For $0<Q_{g} / e<(1+\lambda) / 2$ and $k_{B} T<E_{C}$ one can use the approximation that the system is mainly governed by the two states $n=0$ and $n=1$ (cf. Fig. 4). Generalizing the approach of Sec. (III) the stationary occupation probabilities obey the Boltzmann distribution

$$
\begin{aligned}
& \bar{\sigma}(0)=\frac{e^{-E_{0}^{c h} / k_{B} T}}{e^{-E_{0}^{c h} / k_{B} T}+e^{-\left(E_{1}^{c h}+\delta F(T)\right) / k_{B} T}}, \\
& \bar{\sigma}(1)=\frac{e^{-\left(E_{1}^{c h}+\delta F(T)\right) / k_{B} T}}{e^{-E_{0}^{c h} / k_{B} T}+e^{-\left(E_{1}^{c h}+\delta F(T)\right) / k_{B} T}} .
\end{aligned}
$$

Then $\langle Q\rangle$ is given by

$$
\langle Q\rangle=e \frac{e^{-\left(E_{1}^{c h}\left(Q_{g}\right)+\delta F(T)\right) / k_{B} T}}{e^{-E_{0}^{c h}\left(Q_{g}\right) / k_{B} T}+e^{-\left(E_{1}^{c h}\left(Q_{g}\right)+\delta F(T)\right) / k_{B} T}} .
$$

In Fig. 6 the box charge has been calculated over the main $Q_{g}$-interval for a sample of temperatures using Eq. (13). There are two mechanisms how temperature changes the shape of the charge characteristics. The $k_{B} T$-terms in Eq. (13) make the curve 
smoother but they do not shift the critical values of $Q_{g}$ where the mean charge reach half integer values. This is just reached by the $\delta F(T)$-term. At $\Delta / k_{B} T=8$ corresponding approximately to the cross-over temperature the 2e-periodicity has already disappeared leaving only the $1 \mathrm{e}-$ periodicity. The property $\langle Q\rangle\left(Q_{g}=1 / 2\right)=1 / 2$ means that there are no more even-odd-effects.

But there is another approach to that problem discussing in more detail the transition rates [10]. The transition $n=0$ to $n=1$ is governed by the rate

$$
\Gamma_{1,0}\left(Q_{g}\right)=\frac{1}{e} \operatorname{Im} I_{q_{s}}\left[-\Delta E_{1,0}^{c h}\left(Q_{g}\right) / \hbar\right]\left\{1-e^{\Delta E_{1,0}^{c h}\left(Q_{g}\right) / k_{B} T}\right\}^{-1} .
$$

This corresponds to $\Gamma_{1,0}$ in Eq. (1) for a NIS junction. The current amplitude $I_{q_{s}}$ corresponds to the NIS junction and brings the gap energy $\Delta$ up. But the back rate describing the transition $n=1$ to $n=0$ consists of two terms

$$
\Gamma_{0,1}=\gamma_{0,1}+\Gamma_{0,1}^{(a)}
$$

where the second rate on the right corresponds again to $\Gamma_{0,1}$ in Eq. (4) and reads as

$$
\Gamma_{0,1}^{(a)}\left(Q_{g}\right)=\frac{1}{e} \operatorname{Im} I_{q_{s}}\left[-\Delta E_{0,1}^{c h}\left(Q_{g}\right) / \hbar\right]\left\{1-e^{\Delta E_{0,1}^{c h}\left(Q_{g}\right) / k_{B} T}\right\}
$$

The rate $\gamma_{0,1}$ describes back tunneling of just the one (odd) electron which does not found any partner for pairing from the island and is proportional to $N_{\text {eff }}^{-1}$ [10. This is the difference to the approach of Sec. 2. Of course $\gamma_{0,1}$ depends on the energy differences $\Delta E_{0,1}\left(Q_{g}\right)$ too. The rates are given by "golden rule" integrals [10]

$$
\begin{aligned}
& \Gamma_{1,0}=\frac{2 \pi}{\hbar} \int_{\infty}^{\infty} d \epsilon N_{(n)} N_{(s)}\left(\epsilon-\Delta E_{1,0}^{c h}\right)|\mathcal{T}|^{2} f(\epsilon)\left(1-f\left(\epsilon-\Delta E_{1,0}^{c h}\right)\right), \\
& \left.\gamma_{0,1}=\frac{2 \pi}{\hbar} \int_{\infty}^{\infty} d \epsilon N_{(n)} N_{(s)}\left(\epsilon+\Delta E_{0,1}^{c h}\right)|\mathcal{T}|^{2}(1-f(\epsilon)) \tilde{f}\left(\epsilon+\Delta E_{0,1}^{c h}\right)\right) .
\end{aligned}
$$

$N_{(n)}$ and $N_{(s)}$ are the densities of states of normalconductor and superconductor, respectively. $\mathcal{T}$ is the tunneling amplitude. The distribution $\tilde{f}$ is defined by 


$$
\int_{0}^{\infty} d \epsilon N_{(s)}(\epsilon) \tilde{f}(\epsilon)=1
$$

corresponding to the assumption that there is just one unpaired electron on the island.

Now the mean stationary charge reads as $\left(0<Q_{g} / e<1\right)$

$$
\langle Q\rangle=e \frac{\Gamma_{1,0}\left(Q_{g}\right)}{\gamma_{0,1}+\Gamma_{0,1}^{(a)}\left(Q_{g}\right)+\Gamma_{1,0}\left(Q_{g}\right)} .
$$

What is the connection between Eq. (13) and Eq. (19)? Using Eq. (11), Eq. (13) can be transformed in the following form

$$
\langle Q\rangle=e \frac{e^{-\left(\Delta E_{1,0}^{c h}\left(Q_{G}\right)+\Delta\right) / k_{B} T}}{N_{e f f}^{-1}+e^{-\left(\Delta E_{1,0}^{c h}\left(Q_{g}\right)+\Delta\right) / k_{B} T}} .
$$

This expression is a rather good approximation of Eq. (19) because for $0<Q_{g} / e<$ $(1+\lambda) / 2$ there is

$$
\left.\Gamma_{1,0}\left(Q_{g}\right) \propto \exp \left(-\left(\Delta E_{1,0}^{c h}\left(Q_{g}\right)+\Delta\right) / k_{B} T\right)\right)
$$

and $\Gamma_{0,1}^{(a)} \ll \gamma_{0,1}$. Also for $(1+\lambda) / 2<Q_{g} / e<1$ this formula yields the correct charge value $(\langle Q\rangle / e \rightarrow 1)$. The explicit consideration of the possibility that just the one unpaired electron can tunnel back corresponds to the free energy argument in the other approach.

\section{SUPERCONDUCTING BOX WITH IRRADIATION}

The discussion of the transition rates is necessary because the treatment of irradiation is not possible in the Boltzmann distribution approach. But the rates (14, 15) have to be generalized with respect to arbitrary $n$ and $Q_{g}$.

$$
\begin{aligned}
\Gamma_{n \pm 1, n} & =\gamma_{n \pm 1, n} \cdot P_{n}+\Gamma_{n \pm 1, n}^{(a)} \\
\Gamma_{n \pm 1, n}^{(a)}\left(Q_{g}\right) & =\frac{1}{e} \operatorname{Im} I_{q_{s}}\left[-\Delta E_{n \pm 1, n}^{c h}\left(Q_{g}\right) / \hbar\right]\left\{1-e^{\Delta E_{n \pm 1, n}^{c h}\left(Q_{g}\right) / k_{B} T}\right\}^{-1} .
\end{aligned}
$$

The rate $\gamma$ in Eq. (21) describes either back tunneling of just the one (odd) electron (see Sec. 4) or the direct transition of just the pairing partner of the odd electron on the island. 
The other rates $\Gamma$ describe transitions connected with the production of excited states on the island.

The treatment of the case with microwave irradiation is analogous to that of Sec. 3 . The charge is given by formula (5) where the occupation probabilities $\bar{\sigma}$ are determined by modified transition rates $\bar{\Gamma}$. These modified rates have been phenomenological constructed analogous to Eq. (9)) and read as

$$
\bar{\Gamma}_{\ldots}\left(Q_{g}\right)=\sum_{r} J_{r}^{2}(a) \Gamma_{\ldots}\left(\Delta E_{\ldots}^{c h}\left(Q_{g}\right)+r \hbar \bar{\omega}\right)
$$

where the symbol $\Gamma_{\ldots}$ refers to the rates (21). Now the occupation probabilities $\bar{\sigma}(n)$ have to be calculated. This can be done using standard techniques (see e.g. Ref. [22]). Note that $\sum_{n} \bar{\sigma}(n)=1$. A sample of charge functions with different $\mathrm{AC}$ amplitudes has been plotted in Fig. 7 for fixed temperature.

\section{DISCUSSION}

The investigation shows that the photon assisted tunneling via excited states leads to an AC amplitude dependent cross-over from the $2 \mathrm{e}-$ periodic regime to $1 \mathrm{e}-$ periodicity. The main effect is again due to 1 -photon processes $(r= \pm 1)$. The critical value of $Q_{g}$ where the mean charge reachs the value $1 / 2$, shows that for fixed temperature $T<T^{*}\left(T^{*}\right.$ is the cross-over temperature in case on no irradiation) this value goes with increasing microwave amplitude to $1 / 2$. This means that the cross-over from $2 \mathrm{e}-$ periodic behavior to 1e-periodicity can be reached not only by increasing temperature but also by increasing irradiation amplitude. An analytical formula for the cross-over amplitude cannot be given. The numerical simulation shows that for $T<T^{*}$ the $1 \mathrm{e}$-periodic behavior is already established approximately for $a \approx 0.1$. By increasing the AC amplitude it can also be recognized (cf. Fig. 7) that the mechanism of "smoothing" the staircase function is much more complicated than that of finite temperature. The graphs for medium AC 
amplitudes show kinks at several values $Q_{g}$ which are relics of the kink of the NIS current amplitude at the gap energy.

The master equation approach demands that the rates should be much less than the other relevant frequencies. With respect to the Coulomb frequency $E_{C} / \hbar$ this is in principle guaranteed by $R_{Q} \ll R_{T}$. But this should also be ensured with respect to $\bar{\omega}$. Therefore, we have assumed that $\bar{\omega}$ is approximately of the same order of magnitude as the Coulomb frequency. If the Coulomb energy corresponds approximately to $1 \mathrm{~K}$ the radiation frequency should be in the range of $20 \mathrm{GHz}$. It is reasonable to choose $\bar{\omega}$ in such a way because this yields the natural physical frequency scale and guarantees more clearly effects. Up to now there are no experimental measurements known. On the contrary one tries to suppress photon induced processes by filtering [23].

Acknowledgments: We would like to thank J. Siewert for helpful discussion. For some useful hints we are indepted to D. Vion. This work was supported by the Deutsche Forschungsgemeinschaft. 


\section{REFERENCES}

[1] B. L. Altshuler, P. A. Lee and R. A. Webb, Mesoscopic Phenomena in Solids, Modern Problems in Condensed Matter Sciences (30), (Elsevier, Amsterdam, 1991).

H. Grabert and M. H. Devoret, Single Charge Tunneling: Coulomb Blockade Phenomena in Nanostructures, NATO ASI Series B: Physics, Vol. 294, (Plenum Press, New York and London, 1992).

G. Schön and A. D. Zaikin, Physics Reports 198, 237 (1990).

[2] D. Esteve, Transferring electrons one by one, In: H. Grabert and M. H. Devoret, editors, Single Charge Tunneling: Coulomb Blockade Phenomena in Nanostructures, NATO ASI Series B: Physics, Vol. 294 Plenum Press, New York and London, 1992.

M. H. Devoret, D. Esteve and C. Urbina, Nature 360, 547 (1992).

[3] D. V. Averin and Yu. V. Nazarov, Phys. Rev. Lett. 69, 1993 (1992).

[4] T. S. Tighe M. T. Tuominen, J. M. Hergenrother and M. Tinkham, Phys. Rev. Lett. 69, 1997 (1992).

[5] T. S. Tighe M. T. Tuominen, J. M. Hergenrother and M. Tinkham, Phys. Rev. B 47, 11599 (1993).

[6] P. Lafarge, P. Joyez, D. Esteve, C. Urbina and M. H. Devoret, Phys. Rev. Lett. 70, 994 (1993).

[7] T. M. Eiles, J. M. Martinis and M. H. Devoret, Physica B 189, 210 (1993).

[8] M. T. Tuominen J. M. Hergenrother and M. Tinkham, Phys. Rev. Lett. 72, 1742 (1994).

[9] F. W. J. Hekking, L. I. Glazman, K. A. Matveev and R. I. Shekhter, Phys. Rev. Lett. 70, 4138 (1993). 
[10] G. Schön and A. D. Zaikin, Parity effects on electron tunneling through small superconducting islands, preprint, 1994.

[11] A. Smith, B. Jankó and V. Ambegaokar, BCS superconductivity with fixed number parity, preprint, 1994.

[12] P. Johansson, Phys. Rev. B 41, 9892 (1990).

N. S. Wingreen, A.-P. Jauho and Y. Meir, Phys. Rev. B 48, 8487 (1993).

M. Büttiker, A. Prêtre and H. Thomas, Phys. Rev. Lett. 70, 4114 (1993).

C. Bruder and H. Schoeller, Phys. Rev. Lett. 72, 1076 (1994).

[13] P. K. Tien and J. P. Gordon, Phys. Rev. 129, 647 (1963).

[14] A. A. Odintsov, Fiz. Niz. Temp. 15, 466 (1988).

[15] K. Flensberg, S. M. Girvin, M. Jonson, D. R. Penn and M. D. Stiles, Physica Scripta T42, 189 (1992).

[16] G. Falci, V. Bubanja and G. Schön, Z. Phys. B 85, 451 (1991).

[17] A. Hädicke and W. Krech, Physica B 193, 265 (1993).

[18] D. V. Averin and K. K. Likharev, Zh. Eksp. Teor. Fiz. 90, 733 (1986).

[19] W. Krech, A. Hädicke and H.-O. Müller, Int. J. Mod. Phys. B 6, 3555 (1992).

[20] M. Amman, K. Mullen and E. Ben-Jacob, J. Appl. Phys. 65, 339 (1989).

[21] F. Seume and W. Krech, Ann. Phys. 1, 198 (1992).

[22] H. Grabert, G.-L. Ingold, M. H. Devoret, D. Esteve, H. Pothier and C. Urbina, Z. Phys. B 84, 143 (1991).

[23] D. Vion, P. F. Orfila, P. Joyez, D. Esteve and M. H. Devoret, Miniature electrical filters for single electron devices, preprint, 1994. 


\section{FIGURES}

FIG. 1. Scheme of the considered SET-box

FIG. 2. Charge in the NC box for different temperatures $E_{C} / k_{B} T=100,10,5,1$. The nearly straight line belongs to $E_{C} / k_{B} T=1$.

FIG. 3. Charge in the NC box with irradiation at fixed temperature $E_{C} / k_{B} T=100$ for different $\mathrm{AC}$ amplitudes $a=e V_{1} / \hbar \bar{\omega}=0,0.1,0.5,1, \quad\left(\hbar \bar{\omega} / E_{C}=1\right)$.

FIG. 4. The $E_{n}\left(V_{o}\right)$ parabolas for $\Delta / E_{C}=0.8$. The numbers $n$ of the respective parabolas are given by the $\mathrm{x}$-coordinate of their apices. The lowest crossing points determine the values where charge jumping occurs for $T=0$ (cf. Fig. 5). Note, that for $\Delta / E_{C}>1$ the states with odd number $n$ do not participate; only two-electron tunneling processes (Andreev reflection) take place.

FIG. 5. Charge in the SC box corresponding to the case of Fig. 4 for zero temperature.

FIG. 6. Charge in the SC box in case of $\Delta / E_{C}=0.8$ at different temperatures $\Delta / k_{B} T=8,10,20,50,100, \infty, \quad\left(N(0) \mathcal{V} \Delta \approx 10^{-4}\right.$ corresponding to the sample of Ref. [4]).

FIG. 7. Charge in the SC box with irradiation in case of $\Delta / E_{C}=0.8$ at fixed temperature $\Delta / k_{B} T=100$ for different AC amplitudes $a=e V_{1} / \hbar \bar{\omega}=0,0.01,0.02,0.05,0.1, \quad\left(\hbar \bar{\omega} / E_{C}=1\right.$ , $N(0) \mathcal{V} \Delta \approx 10^{-4}$ corresponding to the sample of Ref. [4]). 